\title{
Desprendimiento de retina regmatógeno pediátrico:
}

\section{una revisión}

\section{Pediatric rhegmatogenous retinal detachment: a review}

\author{
Mario Carranza-Casas*, Mariana López-Martínez, Enrique Ariza-Camacho, Rodrigo Matsui-Serrano, \\ Jorge E. Aceves y Federico Graue-Wiechers \\ Instituto de Oftalmología Conde de Valenciana, Ciudad de México, México
}

\begin{abstract}
Resumen
El desprendimiento de retina regmatógeno en niños y adolescentes es una situación desafiante, pero relativamente rara, que difiere de su contraparte en los adultos en cuanto a características anatómicas, etiología, manejo y pronóstico. En este artículo se destacan las características clínicas y etiopatogénicas, y se realiza una actualización sobre su manejo quirúrgico. El propósito es revisar en detalle dichas características, los factores de riesgo y la técnica de reparación inicial. Se busca proporcionar una base de conocimiento sobre los beneficios, los riesgos y el pronóstico de la cirugía de desprendimiento de retina regmatógeno en niños.
\end{abstract}

Palabras clave: Desprendimiento de retina. Niños. Desprendimiento de retina regmatógeno pediátrico.

\begin{abstract}
Rhegmatogenous retinal detachment in children and adolescents is a challenging but relatively rare disorder that differs from its adult counterpart in terms of anatomical features, etiology, management, and prognosis. In this article, the clinical and etiopathogenic characteristics are highlighted, and an update is made on its surgical management. The aim is to provide a knowledge base on the benefits, risks and prognosis of rhegmatogenous retinal detachment surgery in children.
\end{abstract}

Keywords: Pediatric retinal detachment. Children. Pediatric rhegmatogenous retinal detachment.

\section{Introducción}

El desprendimiento de retina regmatógeno (DRR) en niños y adolescentes es una situación rara, pero desafiante, que difiere de su contraparte adulta en cuanto a características anatómicas, etiología, manejo y pronóstico. El propósito de esta revisión sistemática es repasar los datos clínicos, los factores subyacentes y las técnicas de reparación. Se busca proporcionar una base de conocimiento sobre los beneficios, los riesgos y el pronóstico de la cirugía de DRR en niños, así como comparar los resultados anatómicos y funcionales de los reportes nacionales e internacionales sobre esta patología. Aunque muchos casos tienen resultados insatisfactorios, el manejo cuidadoso e informado puede preservar la visión y mejorar la calidad de vida de estos pacientes.

Correspondencia:

*Mario Carranza Casas

Chimalpopoca 14

Col. Obrera

C.P. 06800, Ciudad de México, México

E-mail: mcc.9105@gmail.com
Fecha de recepción: 08-10-2020

Disponible en internet: 07-09-2021

Fecha de aceptación: 12-05-2021

DOI: 10.24875/RMO.M21000181
Rev Mex Oftalmol. 2021;95(6):267-277

www.rmo.com.mx

0187-4519/๑ 2021 Sociedad Mexicana de Oftalmología. Publicado por Permanyer. Este es un artículo open access bajo la licencia CC BY-NC-ND (http://creativecommons.org/licenses/by-nc-nd/4.0/). 


\section{Método de revisión de la literatura}

Se realizó una revisión de la literatura utilizando la base de datos PubMed. Se realizó una estrategia de búsqueda con términos MeSH usando los operadores booleanos AND y OR de la siguiente manera: ("Pediatric Rhegmatogenous Retinal Detachment" [Mesh] OR "Rhegmatogenous Retinal Detachment Children" [Mesh]) AND "Pediatric Retinal Detachment" [Mesh]. Se obtuvieron en total 68 artículos. La búsqueda se limitó a artículos en inglés publicados desde 1998 hasta agosto de 2020. Se realizó un análisis adicional de la bibliografía en los artículos incluidos y se agregaron las referencias clave, incluyendo datos de un artículo en prensa realizado por los autores de esta revisión. Posterior a la revisión de los resúmenes, se eliminaron artículos repetidos, reportes de caso, cartas al editor, capítulos de libro y artículos que tomaban en cuenta solo una patología asociada (síndrome de Stickler o dermatitis atópica). Asimismo, se eliminaron artículos que trataban principalmente de desprendimientos de retina no regmatógenos o que incluían pacientes mayores de 18 años. Se terminó con un total de 32 documentos.

\section{Definición}

El DRR es la separación de la retina neurosensorial del epitelio pigmentario de la retina causada por un desgarro en esta ${ }^{1}$. El subgrupo pediátrico con esta afección se ha definido con rangos de edad entre el nacimiento y los 18 años ${ }^{1}$. Aunque algunas series toman como límite superior los 15 o 16 años, en general se puede definir como cualquier DRR en menores de 18 años.

\section{Epidemiología}

La incidencia reportada de DRR pediátrico en población general se encuentra en 0.38-0.69 por cada 100,000 habitantes, representando el $0.5-8 \%$ de todos los desprendimientos de retina y el $2-6 \%$ de los regmatógenos $^{1,2}$. La edad media al diagnóstico informada en la literatura varió entre 8.3 y 15.3 años. La literatura reporta de manera consistente una mayor prevalencia en el sexo masculino: entre el $63 \%$ y el $88 \%$ de los desprendimientos se ven en hombres. Esto se explica porque tienen mayor exposición a actividades de riesgo para trauma ocular, que es uno de los principales factores predisponentes para sufrir un $\mathrm{DRR}^{3}$. No se han reportado diferencias en la incidencia entre razas ${ }^{4}$. Las tablas 1-3 muestran los datos epidemiológicos, clínicos y quirúrgicos de manera comparativa entre las series incluidas en esta revisión.

\section{Presentación clínica y evolución natural}

Los DRR en los niños se presentan con peores agudezas visuales, mayor probabilidad de involucro macular y mayores tasas de vitreorretinopatía proliferativa (VRP) en comparación con los adultos ${ }^{5,6}$. La presencia de comorbilidad sistémica y ocular, las características propias del ojo pediátrico, la falta de cooperación para la revisión y la dificultad con el posicionamiento posoperatorio hacen que el manejo sea más complejo ${ }^{5-7}$. A diferencia de los adultos, la progresión suele ser lenta, con pérdida visual subaguda o detectada tardíamente ${ }^{6,7}$. Los niños más pequeños tienen peores resultados debido a la cronicidad del desprendimiento en el momento del diagnóstico, ya que su funcionamiento cognitivo inmaduro dificulta el reconocimiento de los síntomas visuales ${ }^{4,8}$. Una sospecha clínica baja de esta enfermedad y la dificultad de evaluar la retina periférica en esta edad contribuyen al retraso ${ }^{6}$. Esto reafirma la importancia de realizar una adecuada exploración oftalmológica periódica bajo dilatación, en particular en los pacientes con factores de riesgo $0^{1,2}$.

Los niños presentan mayor prevalencia de involucro macular al diagnóstico que los adultos ${ }^{4-10}$, y este es uno de los factores predictores visuales más relevantes en cualquier $\mathrm{DRR}^{3,4}$. Las tasas de afectación macular varían entre el $45.4 \%$ y el $91.4 \%$. El DRR pediátrico también se acompaña de VRP en mayor porcentaje que en los adultos, reportándose entre el $11.4 \%$ y el $46.6 \%$ de los casos. Hay que mencionar que la escala de clasificación utilizada en cada reporte puede diferir y sesgar los resultados. El aumento de la actividad y de la proliferación celular inherente a la edad pediátrica, así como la cronicidad del desprendimiento, son los causantes de la frecuencia de esta complicación y el subsecuente empeoramiento de los resultados quirúrgicos ${ }^{8-11}$. Los desprendimientos bilaterales son también más frecuentes en los niños ${ }^{12}$, encontrándose entre el $2.2 \%$ y el $17.5 \%$ de los casos al momento del diagnóstico. La configuración de los desprendimientos difiere de la que se ve en los adultos, observándose con frecuencia líneas de demarcación, disposición en embudo y macroquistes retinianos que reflejan cronicidad $^{1,2,12}$. Secundario a las razones previamente mencionadas, un alto porcentaje de los desprendimientos en menores de edad, entre el $20 \%$ y el $75 \%$, son totales $^{1,2}$. Es frecuente encontrar desgarros de retina y 
Tabla 1. Datos epidemiológicos y demográficos reportados en los diferentes estudios revisados

\begin{tabular}{|c|c|c|c|c|c|c|c|}
\hline Serie & Pacientes & Ojos & Rango edad, años & Promedio edad, años & Varones & Bilaterales & DRR total \\
\hline Carranza, 2021 & 215 & 242 & $0-18$ & 11.2 & $66 \%$ & $12.5 \%$ & $28.9 \%$ \\
\hline Chen, 2020 & 102 & 112 & $0-18$ & 12.2 & $75 \%$ & $9.8 \%$ & $23.2 \%$ \\
\hline Smith, 2019 & 191 & 212 & $0-18$ & 10.5 & $75 \%$ & $9.9 \%$ & $61 \%$ \\
\hline Tsai, 2018 & 152 & 171 & $0-18$ & 14.2 & $76 \%$ & $12.5 \%$ & NR \\
\hline Read, 2018* & 206 & 231 & $0-15$ & 7.3 & $74 \%$ & $12.1 \%$ & NR \\
\hline McElnea, 2017 & 24 & 28 & $0-16$ & 11.6 & $63 \%$ & $16.6 \%$ & NR \\
\hline Agarkar, 2017 & 9 & 12 & $0-16$ & 12 & $100 \%$ & $33.3 \%$ & $75 \%$ \\
\hline Huang, 2017 & 86 & 86 & $0-18$ & 12.9 & $77 \%$ & $0 \%$ & $25.6 \%$ \\
\hline Fong, 2016 & 47 & 49 & $0-18$ & 13.8 & $60 \%$ & $4.2 \%$ & NR \\
\hline Gurler, 2015 & 29 & 30 & $0-18$ & 12.6 & $79 \%$ & $3.4 \%$ & NR \\
\hline Errera, 2015 & 99 & 104 & $0-18$ & 12 & $80 \%$ & $5.0 \%$ & NR \\
\hline Al-Zaaidi, 2013 & 148 & 166 & $0-16$ & 8.3 & $70 \%$ & $12.1 \%$ & $65.4 \%$ \\
\hline Rahimi, 2013* & 77 & 77 & $0-18$ & 12.5 & $73 \%$ & $0 \%$ & NR \\
\hline Oono, 2012 & 44 & 48 & $0-15$ & 12.3 & $88 \%$ & $9 \%$ & $14.5 \%$ \\
\hline Soheilian, 2009 & 108 & 127 & $0-18$ & 12.1 & $81 \%$ & $17.5 \%$ & NR \\
\hline Wang, 2008 & 107 & 111 & $0-18$ & 13.7 & $75 \%$ & $3.7 \%$ & $20.7 \%$ \\
\hline Gonzales, 2008 & 45 & 46 & $0-18$ & 9.8 & $71 \%$ & $2.2 \%$ & $58 \%$ \\
\hline Wadhwa, 2008 & 216 & 230 & $0-18$ & 11.1 & $82 \%$ & $6.4 \%$ & NR \\
\hline Lee, 2008 & 82 & 88 & $0-16$ & 14 & $73 \%$ & $7.3 \%$ & NR \\
\hline Wang, 2007 & 33 & 33 & $0-18$ & 11 & $76 \%$ & $0 \%$ & $55 \%$ \\
\hline Rumelt, 2006* & 127 & 144 & $0-18$ & 10.8 & $73 \%$ & $13.3 \%$ & NR \\
\hline Chen, 2006 & 32 & 35 & $0-15$ & 12.4 & $75 \%$ & $9.3 \%$ & NR \\
\hline Chang, 2005 & 146 & 152 & $0-18$ & 13.1 & $70 \%$ & $4.1 \%$ & $31.60 \%$ \\
\hline Wang, 2005 & 278 & 296 & $0-18$ & 14.6 & $74 \%$ & $6.4 \%$ & $27.40 \%$ \\
\hline Yokoyama, 2004 & 49 & 55 & $0-15$ & 12 & $86 \%$ & $6.6 \%$ & NR \\
\hline Sarrazin, 2004 & 56 & 60 & $0-18$ & 11.6 & $84 \%$ & $7.1 \%$ & $55 \%$ \\
\hline Weinberg, 2003 & 34 & 39 & $0-18$ & 10.5 & $79 \%$ & $14.7 \%$ & $48.70 \%$ \\
\hline Fivgas, 2001 & 27 & 29 & $0-18$ & 9.6 & $70 \%$ & $7.4 \%$ & NR \\
\hline Akabane, 2001 & 28 & 32 & $0-15$ & 12.8 & $71 \%$ & $14.2 \%$ & NR \\
\hline Haring, 1998 & 31 & 33 & $0-18$ & 15.3 & $55 \%$ & $6.4 \%$ & $3 \%$ \\
\hline
\end{tabular}

degeneraciones retinianas periféricas en el ojo contralateral de estos pacientes, con una prevalencia de hasta el $90 \%{ }^{13}$. Lo anterior aumenta el riesgo de DRR consecutivo, por lo que es vital examinar ambos ojos con énfasis en evaluar la periferia retiniana ${ }^{12}$.

\section{Etiología y factores de riesgo}

La mayoría de los DRR en adultos se asocian a desprendimiento de vítreo posterior, ausente en los casos pediátricos $^{6,7}$. Por lo tanto, estos últimos siempre 
Tabla 2. Datos clínicos y asociaciones causales de los desprendimientos en los diferentes estudios revisados

\begin{tabular}{|c|c|c|c|c|c|c|c|c|}
\hline Serie & Trauma & Miopía & $\begin{array}{l}\text { Definición de } \\
\text { miopía }\end{array}$ & $\begin{array}{l}\text { Anomalías } \\
\text { congénitas }\end{array}$ & $\begin{array}{c}\text { Cirugía intraocular } \\
\text { previa }\end{array}$ & Diálisis & $\begin{array}{l}\text { Involucro } \\
\text { macular }\end{array}$ & VRP \\
\hline Carranza, 2021 & $38.1 \%$ & $34.7 \%$ & $>-1 \mathrm{D}$ & $6 \%$ & $17.8 \%$ & $9 \%$ & $86 \%$ & $43 \%$ \\
\hline Chen, 2020 & EXC & $47.1 \%$ & $>-1 \mathrm{D}$ & $50 \%$ & $11.8 \%$ & $2.7 \%$ & $60.7 \%$ & NR \\
\hline Smith, 2019 & $37 \%$ & $33 \%$ & NR & $23 \%$ & $18 \%$ & $17 \%$ & $91 \%$ & $40 \%$ \\
\hline Tsai, 2018 & $14.6 \%$ & $40.9 \%$ & $>-2 \mathrm{D}$ & $11.1 \%$ & $11.7 \%$ & $1.8 \%$ & $78.9 \%$ & $16.4 \%$ \\
\hline Read, 2018* & $33 \%$ & $29 \%$ & NR & NR & NR & NR & $N R$ & $68 \%$ \\
\hline McElnea, 2017 & $50 \%$ & $40.3 \%$ & NR & NR & NR & $21.4 \%$ & $64.3 \%$ & $42.9 \%$ \\
\hline Agarkar, 2017 & $N R$ & $58.3 \%$ & $\mathrm{LA}>24.5 \mathrm{~mm}$ & NR & $100 \%$ & $0 \%$ & $83.3 \%$ & $16.6 \%$ \\
\hline Huang, 2017 & $27.9 \%$ & $55.8 \%$ & $>-1 \mathrm{D}$ & $39.5 \%$ & $17.4 \%$ & $5.8 \%$ & $75.6 \%$ & $46.6 \%$ \\
\hline Fong, 2016 & $8.5 \%$ & $66.7 \%$ & $>-6 D$ & $17 \%$ & NR & $12.3 \%$ & $57.1 \%$ & $36.7 \%$ \\
\hline Gurler, 2015 & $36.6 \%$ & $40 \%$ & $>-6 \mathrm{D}$ & $6.6 \%$ & NR & $6.6 \%$ & NR & $46.6 \%$ \\
\hline Errera, 2015 & $39 \%$ & $42 \%$ & $>-1 \mathrm{D}$ & $20.1 \%$ & $3.8 \%$ & $53.8 \%$ & $60.5 \%$ & $9.6 \%$ \\
\hline Al-Zaaidi, 2013 & $43 \%$ & $30.7 \%$ & $>-1 D$ & $31 \%$ & $40 \%$ & NR & $78.4 \%$ & $33 \%$ \\
\hline Rahimi, 2013* & $90 \%$ & $6 \%$ & $>-4 \quad D$ & NR & $53 \%$ & $4 \%$ & NR & $14 \%$ \\
\hline Oono, 2012 & $38 \%$ & $41.6 \%$ & $>-3 D$ & $10.4 \%$ & $0 \%$ & $14.5 \%$ & $33.3 \%$ & $15 \%$ \\
\hline Soheilian, 2009 & $44.3 \%$ & $29.1 \%$ & $>-3 D$ & $39.3 \%$ & $7.9 \%$ & $N R$ & $80 \%$ & $45 \%$ \\
\hline Wang, 2008 & $E X C$ & $100 \%$ & $>-6 \mathrm{D}$ & $11.7 \%$ & NR & $5.4 \%$ & $77.4 \%$ & $39.6 \%$ \\
\hline Gonzales, 2008 & $43 \%$ & $17 \%$ & $>-4 \mathrm{D}$ & $21 \%$ & $61 \%$ & $6.5 \%$ & $83 \%$ & $59 \%$ \\
\hline Wadhwa, 2008 & $34 \%$ & $14 \%$ & NR & NR & NR & NR & $98.2 \%$ & $44.8 \%$ \\
\hline Lee, 2008 & $53 \%$ & $17 \%$ & $>-6 \mathrm{D}$ & $27 \%$ & $2.20 \%$ & $44 \%$ & $66 \%$ & $\mathrm{NR}$ \\
\hline Wang, 2007 & $100 \%$ & $N R$ & NR & NR & NR & $9 \%$ & $70 \%$ & $64 \%$ \\
\hline Rumelt, 2006* & $42 \%$ & $11 \%$ & $>-6 \mathrm{D}$ & $36 \%$ & $10 \%$ & $1.40 \%$ & $77 \%$ & $12 \%$ \\
\hline Chen, 2006 & $23 \%$ & $23 \%$ & $>-3 D$ & $34 \%$ & $3 \%$ & NR & $91.4 \%$ & $11.4 \%$ \\
\hline Chang, 2005 & $33 \%$ & $37.5 \%$ & $>-4 \mathrm{D}$ & $11.80 \%$ & $5.90 \%$ & $10.50 \%$ & $73 \%$ & $18.4 \%$ \\
\hline Wang, 2005 & $30.7 \%$ & $38 \%$ & $>-6 \mathrm{D}$ & $17 \%$ & $5 \%$ & $6.80 \%$ & $80 \%$ & $45.6 \%$ \\
\hline Yokoyama, 2004 & $27 \%$ & $25 \%$ & $>-4 \mathrm{D}$ & $26 \%$ & $2 \%$ & $27 \%$ & NR & $22 \%$ \\
\hline Sarrazin, 2004 & $100 \%$ & NR & NR & NR & NR & $3.33 \%$ & $58 \%$ & $3 \%$ \\
\hline Weinberg, 2003 & $36 \%$ & NR & NR & $41 \%$ & $51 \%$ & $5.12 \%$ & $74 \%$ & $31 \%$ \\
\hline Fivgas, 2001 & $E X C$ & $34 \%$ & $>-4 \mathrm{D}$ & $27.60 \%$ & $34 \%$ & $21 \%$ & $79 \%$ & $45 \%$ \\
\hline Akabane, 2001 & $22 \%$ & $38 \%$ & $>-4 \mathrm{D}$ & 18.7 & $9.40 \%$ & $6.30 \%$ & NR & $37.5 \%$ \\
\hline Haring, 1998 & $24 \%$ & $42 \%$ & $>-3 \mathrm{D}$ & NR & $9.10 \%$ & $42.40 \%$ & $45.4 \%$ & NR \\
\hline
\end{tabular}

D: dioptrías; EXC: se excluyeron pacientes con antecedente de trauma; LA: longitud axial; NR: no reportado; VRP: vitreorretinopatía proliferativa. ${ }^{*}$ Se incluyeron algunos casos de otros tipos de desprendimiento de retina.

asocian algún factor causal adicional,14,15. Los casos «idiopáticos» podrían deberse a que este grupo etario no expresa todas las características de las enfermedades comúnmente asociadas, o podría relacionarse con un traumatismo no presenciado. Sin embargo, la evidencia apunta a que en todos los casos existe una etiología subyacente, aunque no sea aparente de manera inicial ${ }^{1,2}$. Los factores etiológicos reportados en las series revisadas pueden mostrar sobreposición entre ellos, ya que muchos pacientes presentan más de un 
Tabla 3. Datos y resultados quirúrgicos de los diferentes estudios revisados

\begin{tabular}{|c|c|c|c|c|c|c|c|c|c|}
\hline Serie & $\begin{array}{l}\text { Duración } \\
\text { de la serie, } \\
\text { años }\end{array}$ & $\begin{array}{c}\text { Ojos } \\
\text { operados }\end{array}$ & $\begin{array}{c}\text { Seguimiento } \\
\text { mínimo, } \\
\text { meses }\end{array}$ & $\begin{array}{c}\text { Promedio de } \\
\text { seguimiento, } \\
\text { meses }\end{array}$ & $\begin{array}{l}\text { Promedio } \\
\text { de cirugías } \\
\text { por ojo }\end{array}$ & $\begin{array}{c}\text { Cirugía } \\
\text { de } \\
\text { cristalino }\end{array}$ & $\begin{array}{c}\text { Éxito } \\
\text { anatómico }\end{array}$ & $\begin{array}{c}\text { Exito } \\
\text { funcional }\end{array}$ & $\begin{array}{l}\text { Definición } \\
\text { de éxito } \\
\text { funcional }\end{array}$ \\
\hline Carranza, 2021 & 16 & 168 & 3 & 51.9 & 1.44 & $22.6 \%$ & $62 \%$ & $54 \%$ & $\geq 20 / 200$ \\
\hline Chen, 2020 & 4 & 112 & NR & NR & NR & NR & NR & NR & NR \\
\hline Smith, 2019 & 14 & 212 & 3 & 36.3 & 1.34 & $20 \%$ & $78 \%$ & NR & NR \\
\hline Tsai, 2018 & 20 & 171 & 6 & 37.8 & 1.22 & NR & $86.7 \%$ & $81.6 \%$ & $\geq 20 / 200$ \\
\hline Read, 2018* & 11 & 178 & 3 & 48 & NR & $38.7 \%$ & $62 \%$ & $25 \%$ & $\geq 20 / 200$ \\
\hline McElnea, 2017 & 8 & 28 & NR & NR & 3.0 & $34.6 \%$ & $84.6 \%$ & $25 \%$ & $\geq 20 / 40$ \\
\hline Agarkar 2017, & 11 & 10 & NR & 45.5 & 1.2 & NR & $83.4 \%$ & $75 \%$ & $\geq 20 / 200$ \\
\hline Huang, 2017 & 5 & 86 & 3 & 46.3 & NR & NR & NR & NR & $\geq 20 / 200$ \\
\hline Fong, 2016 & 12 & 49 & NR & NR & 1.48 & $20.4 \%$ & $85.7 \%$ & NR & NR \\
\hline Gurler, 2015 & NR & 30 & 3 & 8 & 1.2 & NR & $83.3 \%$ & $80 \%$ & $\geq 5 / 200$ \\
\hline Errera, 2015 & 13 & 104 & 3 & 25 & 1.25 & NR & $94 \%$ & NR & NR \\
\hline Al-Zaaidi, 2013 & 10 & 166 & 12 & 40.14 & 1.16 & $51.2 \%$ & $78.3 \%$ & $44 \%$ & $\geq 20 / 200$ \\
\hline Rahimi, 2013* & 5 & 77 & NR & NR & 1.8 & NR & $62.3 \%$ & $51.4 \%$ & $\geq 5 / 200$ \\
\hline Oono, 2012 & 11 & 48 & 3 & 24 & 1.46 & NR & $95.8 \%$ & $85.4 \%$ & $\geq 20 / 200$ \\
\hline Soheilian, 2009 & 8 & 127 & 6 & 34 & 1.55 & $40.1 \%$ & $74.9 \%$ & $37 \%$ & $\geq 20 / 200$ \\
\hline Wang, 2008 & 20 & 111 & 6 & NR & 1.12 & $9.9 \%$ & $90.9 \%$ & $72.9 \%$ & $\geq 20 / 200$ \\
\hline Gonzales, 2008 & 5 & 46 & 3 & 20.4 & NR & $55 \%$ & $78 \%$ & $44 \%$ & $\geq 20 / 200$ \\
\hline Wadhwa, 2008 & NR & 230 & 3 & 12.3 & 1.98 & NR & $88.7 \%$ & $60 \%$ & $\geq 4 / 200$ \\
\hline Lee, 2008 & 15 & 88 & NR & NR & NR & NR & NR & NR & NR \\
\hline Wang, 2007 & 20 & 33 & 6 & 57.1 & 1.45 & $84 \%$ & $36.3 \%$ & $36 \%$ & $\geq 20 / 100$ \\
\hline Rumelt, 2006* & 20 & 144 & 1 & 44.28 & 1.55 & NR & $52 \%$ & $37 \%$ & $\geq 20 / 400$ \\
\hline Chen, 2006 & 9 & 35 & NR & NR & 1.4 & $25.7 \%$ & $80 \%$ & $37.1 \%$ & $\geq 20 / 50$ \\
\hline Chang, 2005 & 14 & 152 & 6 & 48.3 & 1.5 & NR & $78.3 \%$ & NR & NR \\
\hline Wang, 2005 & 20 & 296 & 6 & 51 & 1.34 & $12.1 \%$ & $85 \%$ & $51.7 \%$ & $\geq 20 / 100$ \\
\hline Yokoyama, 2004 & 14 & 55 & 12 & 80 & 1.2 & NR & $87 \%$ & $73 \%$ & $\geq 20 / 200$ \\
\hline Sarrazin, 2004 & 20 & 55 & 1 & 34.8 & NR & NR & $52.7 \%$ & $28 \%$ & $\geq 20 / 200$ \\
\hline Weinberg, 2003 & 9 & 39 & 3 & 31.1 & 1.6 & NR & $79 \%$ & $15 \%$ & NR \\
\hline Fivgas, 2001 & 6 & 29 & 4 & 21.4 & 2.2 & NR & $72 \%$ & $41 \%$ & $\geq 20 / 800$ \\
\hline Akabane, 2001 & 5 & 32 & 6 & 16.4 & 1.1 & NR & $93.8 \%$ & NR & NR \\
\hline Haring, 1998 & 14 & 33 & 3 & 106.8 & 1.15 & NR & $100 \%$ & $60.6 \%$ & $\geq 20 / 40$ \\
\hline
\end{tabular}

factor de riesgo ${ }^{12,14,15}$. Por ejemplo, es más común que Asimismo, es probable que las alteraciones congénitas, un traumatismo cause DRR en niños con longitudes como el síndrome de Stickler, se acompañen de miopía axiales largas 0 antecedente de cirugía intraocular ${ }^{12}$. patológica o cirugías intraoculares previas ${ }^{13}$. 


\section{Trauma}

El factor más prevalente en la mayoría de las series es el trauma ocular ${ }^{1,2}$. Este se encuentra en el $22 \%$ al $53 \%$ de los DRR pediátricos. El desprendimiento en estos casos es generado por un mecanismo indirecto relacionado con la deformación del globo o el daño directo a la pared ocular ${ }^{3,4}$. La formación de desgarros periféricos opuestos al sitio del impacto es el resultado de cambios en la tracción vítrea inducidos por la expansión súbita del ojo ${ }^{3,4}$. En muchos casos se diagnostica el desprendimiento tardíamente, simulando un DRR idiopático $0^{3,4,16}$; esto se debe a la fuerte adherencia vitreorretiniana en pacientes jóvenes y a la ausencia de desprendimiento de vítreo posterior ${ }^{1-4}$. Por otro lado, los DRR traumáticos se asocian a lesiones concomitantes en otras estructuras oculares y VRP, y por consiguiente el pronóstico suele ser pobre ${ }^{3,4,11,16}$. Salvo cuando hay datos de endoftalmitis o de un cuerpo extraño intraocular, la reparación de un DRR postraumático debe realizarse 4 a 7 días después del evento; se busca lograr la mayor licuefacción vítrea antes de intentar inducir un desprendimiento de vítreo posterior durante el procedimiento ${ }^{3,4,6,16}$. La cirugía temprana podría aumentar el riesgo de sangrado debido a la congestión de los tejidos uveales, mientras que un retraso mayor incrementa el riesgo de proliferación fibrovascular y dificulta el manejo ${ }^{7,16}$. Los DRR asociados a trauma penetrante se consideran de peor pronóstico que los causados por una lesión contusa, aunque hay reportes que concluyen lo contrario; una explicación posible es que las ondas de choque indirectas pueden provocar diálisis retiniana y roturas coroideas no detectadas inicialmente, llevando a un DRR crónico de peor resultado 3,4,7,9,14,16.

\section{Miopía}

La miopía es el segundo factor más reportado ${ }^{17}$, encontrado en el $14 \%$ al $58.3 \%$ de los DRR pediátricos. La definición de miopía varía significativamente entre los diferentes estudios (tabla 2) ${ }^{17}$. Este condicionante es más común en los adolescentes, su incidencia aumenta con la edad $d^{4,12,17}$ y su prevalencia también se encuentra en incremento de manera global, principalmente en poblaciones asiáticas ${ }^{4,12,17}$. El mecanismo vinculante de la alta miopía y el desprendimiento retiniano incluye una mayor prevalencia de degeneraciones retinianas periféricas, una bomba coroidea deficiente, un vítreo patológico y el desprendimiento de vítreo posterior temprano ${ }^{14,17}$. Los desprendimientos asociados a miopía en adolescentes probablemente tengan una patogenia y unas características similares a las de los desprendimientos encontrados en los adultos, por lo que en comparación con otras etiologías que cursan con comorbilidad o mecanismos complejos muestran mejores resultados funcionales $4,12-14,17$.

\section{Anomalías congénitas y del desarrollo}

Existen anomalías presentes al nacer o manifiestas durante el desarrollo temprano que predisponen al DRR durante la vida ${ }^{1,2}$. Suelen acompañarse de alteraciones vítreas, incluyendo licuefacción anormal y adhesión patológica del vítreo, así como lesiones predisponentes desde edades tempranas ${ }^{12,18,19}$. La prevalencia de estas anomalías se reporta entre el $6 \%$ y el $39 \%$, y la bilateralidad es la norma ${ }^{19}$. En el caso de los desprendimientos en estos pacientes hay una mayor frecuencia de involucro macular y DRR crónico ${ }^{9,19}$. Se presentan con VRP más grave, peores pronósticos y un mayor número de procedimientos quirúrgicos ${ }^{9,19}$. A continuación se abordan brevemente las condiciones más frecuentemente asociadas a DRR pediátrico.

\section{Síndrome de Stickler}

El síndrome de Stickler es la causa hereditaria más común de DRR en los niños ${ }^{19}$. Existen varios tipos, del I al VI, asociados a mutaciones que codifican para distintos tipos de colágeno ${ }^{1,2}$. El tipo I, que corresponde al $85 \%$ de los diagnósticos, es causado por mutaciones en el gen COL2A1 y se transmite de manera autosómica dominante ${ }^{1,2}$. Esta enfermedad cursa con miopía alta congénita, alto riesgo de DRR, sordera temprana y artropatía generalizada ${ }^{1,2}$. Además, los pacientes presentan formación precoz de catarata, degeneración en encaje periférica y desgarros retinianos hasta en un $90 \%{ }^{1,2}$. El vítreo de estos pacientes es anómalo, ópticamente vacío y con características membranosas retrolentales ${ }^{1,2}$. Mutaciones distintas en el mismo gen o en otras variantes del colágeno pueden provocar fenotipos similares, como la displasia de Kniest y el síndrome de Marshall, que cursan también con alto riesgo de DRR pediátrico ${ }^{1,2}$. El síndrome de Stickler y sus variantes se han asociado a desgarros gigantes como lesiones causales, así como a peor pronóstico funcional tras la reparación ${ }^{13}$.

\section{VitreorRetinopatías asociadas al CROMOSOMA $5 Q$}

La enfermedad de Wagner, la enfermedad de Jansen y la vitreorretinopatía erosiva son afecciones dentro de 
un mismo espectro patológico causadas por mutaciones autosómicas dominantes en el gen CSPG2/ $\operatorname{VCAN}^{1,2}$. El gen afectado está localizado en el cromosoma 5 y codifica para el proteoglicano versican de la matriz extracelular, involucrado en el mantenimiento de la estructura vítrea ${ }^{1,2}$. Dichas enfermedades se caracterizan por miopía moderada y degeneración vítrea con velos avasculares ecuatoriales ${ }^{1,2}$. Se asocian a DRR en la adolescencia, por lo que pueden encontrarse en las series de DRR pediátrico ${ }^{1,2}$.

\section{Síndrome de Marfan}

Síndrome autosómico dominante en el que se afecta el gen que codifica la fibrilina, proteína que participa en el andamiaje de la elastina ${ }^{1,2}$. Sus hallazgos sistémicos incluyen extremidades largas, aracnodactilia, articulaciones hiperextensibles y defectos vasculares ${ }^{1,2}$. La manifestación ocular más común es la subluxación del cristalino, presente en el $50-80 \%$ de los afectados ${ }^{1,2}$. La miopía axial también es frecuente: más del $20 \%$ presentan miopías mayores de 7 dioptrías ${ }^{1,2}$. La propia miopía y las altas tasas de cirugía intraocular determinan una mayor prevalencia de DRR pediátrico $0^{1,2}$.

\section{Retinosquisis JUVENIL LIGADA al X}

Es una enfermedad ligada al cromosoma $X$ (encontrada predominantemente en varones) que se caracteriza por estrías radiales en la mácula secundarias a esquisis foveal, así como por la separación periférica de la retina ${ }^{1,2}$. Está causada por mutaciones en el gen RS1 que codifica la retinosquisina, proteína esencial en la adhesión celular entre fotorreceptores y células bipolares $^{1,2}$. La esquisis foveal cursa con baja visual central, pero es la esquisis periférica la que puede llegar a causar DRR en el $5 \%$ al $22 \%$ de los $\operatorname{casos}^{1,2,14}$. La reparación de estos desprendimientos se complica por una hialoides firmemente adherida a la pared interna de la retina desprendida ${ }^{1,2}$.

\section{Cirugía intraocular previa (pseudofaquia o afaquia)}

Cualquier cirugía intraocular implica alteración y tracción del vítreo, así como un riesgo de generar desprendimiento de vítreo posterior; esto condiciona una predisposición a padecer $\mathrm{DRR}^{6,7}$. Por otra parte, los pacientes afáquicos pierden el soporte de la base vítrea, lo cual aumenta el riesgo de múltiples y pequeños desgarros anteriores, particularmente difíciles de identificar en niños ${ }^{7}$. La literatura reporta una prevalencia variable, entre el $2 \%$ y el $61 \%$. Un gran porcentaje corresponde a niños operados de catarata congénita 0 por glaucoma del desarrollo 4 ; la comorbilidad y las complicaciones encontradas en estos casos conllevan peores pronósticos visuales y anatómicos ${ }^{7-9}$.

\section{Retinopatía del prematuro}

Aunque los pacientes con retinopatía del prematuro activa suelen cursar con desprendimientos traccionales, los que muestran regresión de esta patología pueden presentan altas tasas de desprendimiento regmatógeno de manera relativamente tardía ${ }^{7}$. Esto se debe a un riesgo elevado de desarrollar miopía alta, lesiones predisponentes y alteraciones vítreas ${ }^{1,20}$. La fisiopatogenia de estos cambios no está aclarada ${ }^{1,20}$. Los casos de DRR con antecedentes de parto pretérmino y retinopatía del prematuro tienen mayor tasa de VRP y de desprendimiento total, que se acompañan de peores resultados visuales ${ }^{1,20,21}$.

\section{Diálisis retiniana del adulto joven}

La diálisis retiniana es un tipo específico de desgarro retiniano orientado circunferencialmente, ubicado anterior a la ora serrata dentro del epitelio ciliar, o posterior a la ora dentro de la retina ${ }^{1,2}$. La causa más común es el trauma ${ }^{8,18}$, pero también se ha descrito un subgrupo de aparente origen genético ${ }^{15}$. Este último es conocido como diálisis retiniana del adulto joven o diálisis idiopática, y se cree que se origina a partir de la degeneración quística periférica ${ }^{15}$. Es más común en los varones y tiende a encontrarse en la retina temporal de ojos emétropes durante la segunda o tercera décadas de la vida $^{15}$. Los desprendimientos secundarios a diálisis progresan lentamente y se acompañan de líneas de demarcación, evidenciando cronicidad ${ }^{1,2,15}$. La VRP es poco común y tiene buenos resultados con cerclaje escleral ${ }^{15}$.

\section{Coloboma coriorretiniano}

Los colobomas coriorretinianos son anomalías congénitas causadas por una falla en el cierre de la fisura embrionaria. Se caracterizan por una ausencia sectorial de retina, epitelio pigmentario de la retina y coroides ${ }^{18}$. Pueden presentarse aislados o asociados con otras alteraciones, especialmente colobomas de otras capas oculares $^{1,2}$. Pueden ser unilaterales o bilaterales, y típicamente se ubican en el cuadrante inferotemporal ${ }^{18}$. 
Pueden involucrar la mácula, acompañándose entonces de pobre función visual ${ }^{1,2}$. Es común que se compliquen con desgarros retinianos en sus bordes, lo que puede llevar a $\mathrm{DRR}^{18}$. Su ubicación posterior suele requerir vitrectomía ${ }^{18}$.

\section{Dermatitis atópica}

Se ha descrito una asociación entre la dermatitis atópica y los $\mathrm{DRR}$ pediátricos únicamente en pacientes asiáticos ${ }^{8,9,22}$. La teoría principal se basa en que el trauma crónico secundario al tallado ocular recurrente puede ocasionar alteraciones vítreas, diálisis retiniana y lesiones predisponentes que llegan a provocar un DRR $^{8-9,22 .}$.

\section{Otros}

Se han informado casos de DRR pediátrico en relación con otras patologías ${ }^{1-10}$. Por la rareza de estos reportes, no se puede confirmar de manera definitiva su asociación causal. Incluyen anomalía de Morning Glory, rubeola congénita, infección congénita por citomegalovirus, toxoplasmosis congénita, toxocariasis, pars planitis, necrosis retiniana aguda, retinoblastoma, enfermedad de Coats, síndrome de Sturge-Weber y aniridia. Las comúnmente mencionadas asociaciones entre buftalmos, microftalmos y retraso mental con DRR probablemente se deban a que estas condiciones suelen estar acompañadas de otras de las anomalías congénitas descritas 0 de cirugía intraocular previa $^{1-10}$

\section{Evaluación y diagnóstico}

\section{Historia clínica}

La evaluación de los pacientes pediátricos con DRR debe incluir una anamnesis detallada. Las presentaciones más comunes son la baja visual y la leucocoria; el estrabismo y la pobre fijación se han reportado de manera menos frecuente ${ }^{18}$. Se deben investigar los factores predisponentes referidos previamente y los posibles síndromes hereditarios en la historia fami$\operatorname{liar}^{1,2}$. El antecedente de trauma ocular debe ser investigado, ya que podría relacionarse con el tiempo de evolución del DRR, que es un indicador del pronóstico

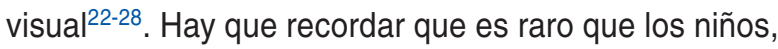
y especialmente los menores de 6 años, refieran síntomas visuales ${ }^{14,15}$.

\section{Exploración}

La agudeza visual y la refracción son datos básicos en la exploración del paciente pediátrico con DRR. Si es necesario, en los niños preverbales, la evaluación de fondo de ojo bajo dilatación se realizará en el quirófano bajo anestesia general por la falta de cooperación. Ante la presencia de opacidad de medios o pobre dilatación, se puede utilizar al ultrasonido como método diagnóstico para confirmar el desprendimiento u observar otros hallazgos relevantes ${ }^{1,2}$.

Es común encontrar lesiones en el ojo contralateral (hasta en el $90 \%$ de los $\operatorname{casos}^{13}$ ) y muchas veces se diagnostica como hallazgo un desprendimiento de esta manera ${ }^{18}$; por lo tanto, es vital revisar ambos ojos. El tipo de lesión causal es variable, y es común no poder identificar la causa debido a la dificultad para realizar un examen adecuado. Se ha descrito como lesión más frecuente el desgarro en herradura ${ }^{13,23}$, pero las series coinciden en que las degeneraciones en encaje asociadas o no a agujeros atróficos son las lesiones pre-

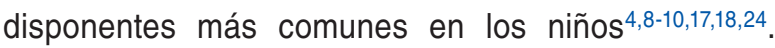
Estas últimas son especialmente frecuentes en pacientes miopes, y van de la mano con un menor riesgo de desarrollar VRP 9 . En los pacientes con antecedente de trauma deben buscarse intencionadamente diálisis retiniana, desgarros gigantes o avulsión de la base vítrea ${ }^{3}$. La búsqueda de signos sistémicos de enfermedades hereditarias es también indispensable. Las anomalías del tronco y de las extremidades, las anomalías faciales, la hipoacusia y el retraso cognitivo, entre otros, podrían indicar la presencia de algún síndrome $e^{1,2}$.

\section{Manejo, resultados y pronóstico}

El tratamiento es quirúrgico en la mayoría de los casos $^{1-2,26}$ y los resultados varían según la comorbilidad, la etiología, la cronicidad y el procedimiento inicial25-28; sin embargo, son menos satisfactorios en comparación con los adultos ${ }^{18,19}$. En un paciente que ya cuenta con antecedente, el riesgo de DRR en el ojo contralateral es aproximadamente del $10 \%^{24,25}$.

\section{Técnicas quirúrgicas}

La reparación en los DRR pediátricos sigue principios similares a los descritos en los adultos, incluyendo la eliminación de la tracción vítrea, el drenaje del líquido subretiniano y la retinopexia con láser o crioterapia ${ }^{25-28}$. Sin embargo, existen diferencias anatómicas y funcionales en el ojo pediátrico que exigen 
consideraciones específicas en el momento de elegir la técnica quirúrgica ${ }^{25-28}$.

Los abordajes iniciales disponibles incluyen el cerclaje escleral, la vitrectomía vía pars plana (VPP) o la combinación de ambas ${ }^{25-28}$.

El cerclaje escleral primario sigue siendo utilizado en muchos casos como el procedimiento inicial cuando hay una adecuada visualización retiniana y ausencia de VRP significativa ${ }^{8-10,26}$. Su beneficio se encuentra en ser una cirugía extraocular que no implica manipulación del vítreo y además evita la necesidad de reintervenir al paciente cuando se utiliza tamponade con silicón $n^{17,18,26}$. En la literatura revisada, el cerclaje escleral primario se realizó en un número variable de casos, entre el $12 \%$ y el $86 \%$. Esta discrepancia corresponde a los diferentes factores etiológicos que se incluyeron en cada estudio ${ }^{26}$. Diversos estudios han reportado excelentes resultados con el uso del cerclaje, particularmente en DRR asociados a miopía ${ }^{17,26,28-30}$. La eficacia del cerclaje escleral primario en estos casos puede deberse a un efecto de tamponade del vítreo no licuefacto presente en un ojo joven al relajarse la tracción con el cerclaje. Esta ventaja también podría corresponder a un sesgo, ya que dicha técnica se prefiere en desprendimientos menos complicados ${ }^{18,26}$. Generalmente son pacientes fáquicos, que no presentan desgarros posteriores ni VRP significativa ${ }^{25-28}$. Esta última es la causa más frecuente de fallo de una cirugía epiescleral18,26.

El cerclaje escleral se ha realizado de manera tanto segmentaria ${ }^{8,25-26}$ como circunferencial ${ }^{4,16,17,25-26}$, y las elecciones de los métodos y de los materiales utilizados varían de acuerdo con la naturaleza del desprendimiento, el tamaño del paciente y la preferencia del cirujano ${ }^{25,26}$. No hay un consenso sobre la preferencia de la localización, el estilo o el tamaño del exoplante, ni sobre la indicación del exodrenaje ${ }^{19,25,26}$.

La VPP ha ido aumentando en popularidad y es la técnica preferida en casos de desprendimientos complejos, totales, con VRP significativa, opacidad de medios, lesiones posteriores, membranas epirretinianas o colobomas coriorretinianos ${ }^{17-19,23-26}$. Los pacientes en quienes no se puede visualizar adecuadamente la retina de manera preoperatoria suelen requerir vitrectomía ${ }^{25-28}$. Los DRR postraumáticos y los asociados a anomalías congénitas suelen ser manejados con $\mathrm{VPP}^{3}$, que es también el procedimiento de elección en casos recidivantes y en pacientes con cirugía intraocular previa ${ }^{25-28}$. Sin embargo, se prefiere evitar la VPP dentro de lo posible, por varias razones. En primer lugar, en los niños, el vítreo es menos licuefacto y la hialoides posterior se encuentra fuertemente adherida a la superficie retiniana ${ }^{8,17,23-28}$. La complejidad de su separación aumenta la dificultad quirúrgica y predispone a desgarros iatrogénicos; es común que se tenga que recurrir a las retinectomías, ya que la fuerza de la interfaz vitreorretiniana pediátrica puede impedir liberar por completo la tracción retiniana ${ }^{23-28}$. En segundo lugar, la VPP se relaciona con el desarrollo de catarata secundaria, cuya morbilidad en este grupo etario es significativa por el alto riesgo de ambliopía y la pérdida de la acomodación asociada a su resolución quirúrgica $^{23-28}$. Finalmente, se ha descrito un mayor riesgo de nuevo desprendimiento y de VRP en los pacientes que se sometieron a VPP22; esto puede reflejar simplemente la gravedad de los desprendimientos en los que se escoge la vitrectomía más que una influencia negativa de esta ${ }^{4,16,17}$. En la mayoría de los estudios se recomienda realizar una vitrectomía de tres puertos 25-28. Los puertos de entrada se colocaron a través del limbo, la pars plicata o la pars plana, según la edad del niño, el tamaño del ojo y la naturaleza del desprendimiento ${ }^{25-28}$. Durante la cirugía debe inducirse el desprendimiento de vítreo posterior, y secundario a este es imperativo retirar de la manera más completa la hialoides posterior para minimizar la posibilidad de contracción vítrea posoperatoria que pueda provocar una recidiva, inducción de VRP o formación de membranas epirretinianas ${ }^{25-28}$. El vítreo no desprendido puede ser difícil de visualizar, y el acetónido de triamcinolona se ha utilizado para facilitar la identificación de la hialoides ${ }^{22-28}$. En casos de catarata traumática está indicada la lensectomía ${ }^{1,2}$.

En algunos casos, la VPP combinada con cerclaje escleral ha mostrado mayores tasas de éxito que la VPP sola ${ }^{21}$. El cerclaje da soporte a la base vítrea y reduce las tracciones residuales causadas por restos del vítreo cerca del desgarro ${ }^{21-28}$. Debido a las características rígidas del vítreo pediátrico, este mismo puede contraerse y llevar a la recurrencia ${ }^{21-28}$. Relajar dichas tracciones facilita la reabsorción del líquido subretiniano, posiblemente reduciendo el riesgo de recidiva y mejorando el pronóstico ${ }^{21-28}$. Hay mayor evidencia de la ventaja del procedimiento combinado en casos con opacidad de medios, desgarros gigantes o múltiples, desprendimientos posteriores y VRP significativa ${ }^{18}$.

La elección del taponamiento en niños es controversial. Aunque se ha descrito el uso de perfluoropropano $4,16,17$, considerando la posibilidad del posicionamiento posoperatorio inadecuado, y los picos de presión intraocular asociados al taponamiento con 
gas, en los niños se prefiere el aceite de silicón. Este último está indicado en casos de reoperación y VRP extensa ${ }^{4,9,16,19}$. Se han documentado malos resultados asociados al uso de silicón como taponamiento, aunque esto probablemente refleja la gravedad de los desprendimientos en los que se eligió realizarlo más que un efecto negativo directo de su aplicación ${ }^{11}$. Se recomienda retirar el silicón después de un mínimo de 3 meses con el enfoque de rehabilitación visual y recordando las complicaciones de su uso, como el riesgo de glaucoma y de queratopatía en banda ${ }^{18}$.

\section{Resultados}

El éxito anatómico (definido como una retina completamente aplicada en ausencia de taponamiento con silicón) se logró en más del $60 \%$ de los pacientes en

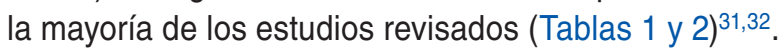
Debido a la dificultad en la valoración visual y refractiva adecuada en niños menores de 6 años, el resultado anatómico toma relevancia importante al tener una fuerte correlación con la capacidad visual a largo plazo $4,16,17$. Las características clínicas que pudieron predecir el éxito anatómico son la ausencia de involucro macular y la subtotalidad del desprendimiento ${ }^{4,7,9,16,17}$. Asimismo, se encontraron mejores resultados anatómicos en los ojos con miopía como factor etiológico $0^{9}$. De manera contraria, la presencia de VRP significativa y el antecedente de cirugía intraocular fueron predictores de fallo $0^{4,7-10}$.

Es difícil reportar de manera confiable y comparable los resultados visuales en pacientes pediátricos, y el éxito visual se definió de manera variable entre las series. En los estudios incluidos, la definición más común de éxito funcional fue una agudeza mejor corregida en la última revisión $\geq 20 / 200$ (Snellen). Entre los grupos que utilizaron esta definición, el promedio de éxito visual fue del $54 \%$.

Al evaluar el éxito visual, la ausencia de involucro macular, la subtotalidad del DRR y la miopía asociada fueron predictores positivos. De manera contraria, los predictores de un pobre resultado fueron una mala visión preoperatoria, una VRP significativa, la cirugía intraocular previa, el desprendimiento total, el trauma penetrante, las anomalías congénitas, la menor edad en el momento del diagnóstico, la necesidad de vitrectomía y el uso de aceite de silicón ${ }^{4,8-11,16,17}$.

La revisión bibliográfica revela que la edad es un condicionante del resultado ${ }^{26-28}$. Los niños más pequeños tienen peor pronóstico visual debido a la mayor frecuencia de anomalías congénitas ${ }^{4,7}$, retinopatía del prematuro o trauma penetrante como etiología ${ }^{9,26}$. El diagnóstico tardío en ellos se refleja en unas mayores tasas de involucro macular, desprendimiento total y VRP significativo ${ }^{4,16-17,26}$. Los niños mayores tienen menor prevalencia de involucro macular y, por lo tanto, presentan mejor pronóstico ${ }^{14}$.

Los resultados quirúrgicos varían también dependiendo de la etiología, como ya se ha mencionado en los apartados dedicados a los factores predisponentes. Aunque algunos estudios lo han puesto en $\mathrm{duda}^{27}$, existe coincidencia en la literatura respecto a los mejores resultados encontrados en DRR relacionados con miopía ${ }^{4,12-14,16,17}$. Las causas de esta correlación incluyen que en ellos se encuentra un vítreo relativamente sano en comparación con otras etiologías como el trauma y las anomalías congénitas ${ }^{7-9,14,19}$. Otra explicación argumenta que en los ojos miopes hay menores concentraciones de citocinas y de factores de crecimiento dentro del epitelio pigmentario de la retina ${ }^{14,27}$. Es probable que estos pacientes representen un subgrupo que responde adecuadamente al cerclaje escleral primario ${ }^{14,24}$. Además, es importante mencionar la asociación entre la miopía y el DRR en los niños mayores $^{4,16,17}$. Lo anterior podría ser una variable de confusión, puesto que la evaluación visual es más confiable en estos últimos, hay mayor cooperación en el manejo posoperatorio y, como ya se ha mencionado, tienden a presentar mejores resultados ${ }^{4,16,17}$.

En los pacientes pediátricos siempre existe un alto riesgo de ambliopía, incluso cuando se logra el éxito anatómico. Por lo tanto, algunos autores sugieren la oclusión posoperatoria en todos los pacientes menores de 10 años, así como una rehabilitación visual y una corrección refractiva realizadas de la manera más intensiva posible $3-4,16,17$.

\section{Conclusiones}

En comparación con los DRR en los adultos, en los niños tienen etiologías distintas y difieren en su presentación, características y pronóstico. La reparación plantea desafíos únicos para el cirujano debido a sus características, así como a la anatomía y la fisiología del ojo pediátrico. Si bien los resultados visuales son pobres en comparación con los de la población adulta, la alta tasa de desprendimientos bilaterales y patología en el ojo contralateral hacen que sea de suma importancia poder reconocer y tratar a estos niños de manera temprana.

En el futuro, nuevos enfoques e instrumentos quirúrgicos, tratamientos alternativos y coadyuvantes como 
la terapia con células madre, la terapia de reemplazo génico, el trasplante de retina y el desarrollo de nuevas prótesis retinianas, junto con los avances en ayudas visuales, podrán mejorar los resultados funcionales de estos niños.

\section{Financiación}

No se requirió financiación para este artículo, la investigación se realizó con los propios medios de los autores.

\section{Conflicto de intereses}

Los autores no tienen ningún conflicto de interés para reportar.

\section{Responsabilidades éticas}

Protección de personas y animales. Los autores declaran que para esta investigación no se han realizado experimentos en seres humanos ni en animales.

Confidencialidad de datos. Los autores declaran que han seguido los protocolos de su centro de trabajo sobre la publicación de datos de pacientes.

Derecho a la privacidad y consentimiento informado. Los autores han obtenido el consentimiento informado de los pacientes y/o sujetos referidos en el artículo. Este documento obra en poder del autor de correspondencia.

\section{Bibliografía}

1. Nuzzi R. Lavia C, Spinetta R. Paediatric retinal detachment: a review. Int J Ophthalmol. 2017;10):1592-03.

2. Wenick AS, Barañano DE. Evaluation and management of pediatric rhegmatogenous retinal detachment. Saudi J Ophthalmol. 2012;26:255-63.

3. Sarrazin L, Averbukh E, Halpert M, Hemo I, Rumelt S. Traumatic paediatric retinal detachment: a comparison between open and closed globe injuries. Am J Ophthalmol. 2004;137:1042-49.

4. Wang NK, Tsai CH, Chen YP, Yeung L, Wu WC, Chen TL, et al. Paediatric rhegmatogenous retinal detachment in East Asians. Ophthalmology. 2005;112:1890-5.

5. Häring $\mathrm{G}$, Wiechens $\mathrm{B}$. Long-term results after scleral buckling surgery in uncomplicated juvenile retinal detachment without proliferative vitreoretinopathy. Retina. 1998;18:501-5

6. Rumelt S, Sarrazin L, Averbukh E, Halpert M, Hemo I. Paediatric vs adult retinal detachment. Eye (Lond). 2007;21:1473-8.

7. Gonzales CR, Singh S, Yu F, Kreiger AE, Gupta A, Schwartz SD. Pediatric rhegmatogenous retinal detachment: clinical features and surgical outcomes. Retina. 2008;28:847-52.
8. Yokoyama T, Kato T, Minamoto A, Sugihara A, Imada M, Kuwabara R, et al. Characteristics and surgical outcomes of paediatric retinal detachment. Eye (Lond). 2004;18:889-92.

9. Chang PY, Yang CM, Yang CH, Huang JS, Ho TC, Lin CP, et al. Clinical characteristics and surgical outcomes of paediatric rhegmatogenous retinal detachment in Taiwan. Am J Ophthalmol. 2005;139:1067-72.

10. Akabane N, Yamamoto S, Tsukahara I, Ishida M, Mitamura Y, Yamamoto $\mathrm{T}$, et al. Surgical outcomes in juvenile retinal detachment. Jpn J Ophthalmol. 2001;45:409-11.

11. Weinberg DV, Lyon AT, Greenwald MJ, Mets MB. Rhegmatogenous retinal detachments in children: risk factors and surgical outcomes. Ophthalmology. 2003;110:1708-13.

12. Chen $\mathrm{SN}$, Jiunn-Feng $\mathrm{H}$, Te-Cheng $\mathrm{Y}$. Pediatric rhegmatogenous retinal detachment in Taiwan. Retina. 2006;26:410-4.

13. Fivgas GD, Capone A Jr. Pediatric rhegmatogenous retinal detachment. Retina 2001;21:101-6.

14. Huang YC, Chu YC, Wang NK, Lai CC, Chen KJ, Hwang YS, et al. Impact of etiology on the outcome of pediatric rhegmatogenous retinal detachment. Retina. 2019;39:118-26.

15. Lee RW, Mayer EJ, Markham RH. The aetiology of pediatric rhegmatogenous retinal detachment: 15 years experience. Eye (Lond). 2008;22:636-40.

16. Wang NK, Chen YP, Yeung L, Chen KJ, Chao AN, Kuo YH, et al. Traumatic pediatric retinal detachment following open globe injury. Ophthalmologica. 2007;221:255-63.

17. Wang NK, Chen YP, Lai CC, Chen TL, Yang KJ, Kuo YH, et al. Paediatric retinal detachment: comparison of high myopia and extreme myopia. Br J Ophthalmol. 2009:93:650-5.

18. Wadhwa N, Venkatesh P, Sampangi R, Garg S. Rhegmatogenous retinal detachments in children in India: clinical characteristics, risk factors, and surgical outcomes. J AAPOS. 2008;12:551-4.

19. Soheilian M, Ramezani A, Malihi M, Yaseri M, Ahmadieh H, Dehghan MH, et al. Clinical features and surgical outcomes of pediatric rhegmatogenous retinal detachment. Retina. 2009;29:545-51.

20. Read SP, Aziz HA, Kuriyan A, Kothari N, Davis JL, Smiddy WE, et al. Retinal detachment surgery in a pediatric population. Retina. 2018;38:1393-402.

21. Smith JM, Ward LT, Townsend JH, Yan J, Hendrick AM, Cribbs BE, et al. Rhegmatogenous retinal detachment in children: clinical factors predictive of successful surgical repair. Ophthalmology. 2019;126:1263-70.

22. Oono $Y$, Uehara K, Haruta M, Yamakawa R. Characteristics and surgical outcomes of paediatric rhegmatogenous retinal detachment. Clin Ophthalmol. 2012;6:939-43.

23. Rahimi M, Bagheri M, Nowroozzadeh MH. Characteristics and outcomes of paediatric retinal detachment surgery at a tertiary referral center. J Ophthalmic Vis Res. 2014:9:210-4.

24. Agarkar S, Gokhale VV, Raman R, Bhende M, Swaminathan G, Jain M. Incidence, risk factors, and outcomes of retinal detachment after pediatric cataract surgery. Ophthalmology. 2018;125:36-42.

25. Al-Zaaidi S, Al-Rashaed S, Al-Harthi E, Al-Kahtani E, Abu El-Asrar AM Rhegmatogenous retinal detachment in children 16 years of age or younger. Clin Ophthalmol. 2013;7:1001-4.

26. Errera MH, Liyanage SE, Moya R, Wong SC, Ezra E. Primary scleral buckling for paediatric rhegmatogenous retinal detachment. Retina. 2015;35:1441-9.

27. Gurler B, Coskun E, Öner V, Comez A, Erbagci I. Clinical characteristics and surgical outcomes of paediatric rhegmatogenous retinal detachment. Int Ophthalmol. 2016;36:521-5.

28. McElnea E, Stephenson K, Gilmore S, O'Keefe M, Keegan D. Paediatric retinal detachment: aetiology, characteristics and outcomes. Int J Ophthalmol. 2018;11:262-6.

29. Tsai A, Wong CW, Lim L, Yeo I, Wong D, Wong E, et al. Pediatric retinal detachment in an Asian population with high prevalence of myopia. Retina. 2019;39:1751-60.

30. Chen C, Huang S, Sun L, Li S, Huang L, Wang Z, et al. Analysis of etiologic factors in pediatric rhegmatogenous retinal detachment with genetic testing. Am J Ophthalmol. 2020;218:330-6.

31. Fong A, Yip P, Kwok T, Tsang CW. A 12-year review on the aetiology and surgical outcomes of paediatric rhegmatogenous retinal detachments in Hong Kong. Eye. 2016;30:355-61.

32. Carranza-Casas M, Quiroz-Gonzalez E, Hernández-Reyes A, Matsui-Serrano R, Ariza-Camacho E, Graue-Wiechers F. Pediatric rhegmatogenous retinal detachment: predictors of anatomic and functional success. Int Ophthalmol. 2021 Apr 11. doi: 10.1007/s10792-021-01834-w. Online ahead of print. 